Arthroskopie $2018 \cdot 31: 111-116$ https://doi.org/10.1007/s00142-017-0176-4 Online publiziert: 7. November 2017 (c) Der/die Autor(en) 2017. Dieser Artikel ist eine Open-Access-Publikation.

Markus Walther · Anke Röser · Hubert Hörterer · Andreas Töpfer · Alexander Mehlhorn · Oliver Gottschalk

Zentrum für Fuß- und Sprunggelenkchirurgie, Schön Klinik München Harlaching, FIFA Medical Centre, München, Deutschland

\title{
Autologe matrixinduzierte Chondrogenese am Sprunggelenk
}

\section{Indikationen} ter Evidenz durch die Mikrofrakturierung therapiert [19, 22]. Allerdings verschlechtern sich die Ergebnisse der Mikrofrakturierung nach etwa 5 Jahren [7, 8]. Auch sind die Ergebnisse bei Defekten $>1,5 \mathrm{~cm}^{2}$ nach Mikrofrakturierung schlechter als bei kleinen Defekten [17]. Gleiches gilt für die Mikrofrakturierung in stark belasteten Zonen wie der Taluskante.

Das Ziel der autologen matrixinduzierten Chondrogenese (AMIC) ist es, den durch die Mikro- oder Nanofrakturierung freigesetzten Stammzellen eine bessere biologische Umgebung zu bieten. Durch eine Kollagenmembran kann der Superclot stabilisiert werden, was die Stammzellen an der gewünschten Lokalisation hält [24]. Verschiedene Arbeiten konnten zeigen, dass durch eine Kollagenmembran die Differenzierung der Stammzellen in Richtung Chondrozyten unterstützt wird [23]. Trotzdem kann auch mit dieser Technik kein hyaliner Knorpel erreicht werden. Allerdings ist die Differenzierung der Stammzellen in Richtung Chondrozyten in einer Kollagenmembran anhand von biologischen Markern nur marginal von den Zellen zu unterscheiden, die nach einer wesentlich aufwändigeren und kostenintensiveren Chondrozytentransplantation (ACI) gefunden werden [13]. Entsprechende Untersuchungen wurden am Tiermodell durchgeführt, vergleichende Studien am Menschen stehen bisher allerdings aus.
Die Indikation zur matrixinduzierten Chondrogenese besteht immer dann, wenn durch eine Mikrofakturierung wahrscheinlich kein zufriedenstellendes Ergebnis zu erreichen ist.

Dies betrifft v. a. Läsionen an der Taluskante mit hoher mechanischer Belastung sowie Defekte mit einer Größe von mehr als $1,5 \mathrm{~cm}^{2}$, wobei einige nordamerikanische Arbeitsgruppen die Grenze bei $1,0 \mathrm{~cm}^{2}$ sehen [15]. Weitere Indikationen sind fehlgeschlagene Mikrofakturierung, Revisionen nach OATS ${ }^{\circledR}$ („osteochondral autograft transfer system") oder auch traumatische Knorpelschäden im Rahmen von Frakturversorgungen.

\section{Operativer Zugang mit Innenknöchelosteotomie}

Die über die letzten Jahre am häufigsten durchgeführte Operationstechnik ist die Knorpelrekonstruktion über eine Osteotomie des medialen Malleolus. Der Innenknöchel ermöglicht eine sehr gute Visualisierung der medialen Taluskante und damit auch eine exzellente Versorgung von osteochondralen Läsionen in diesem Bereich [33]. Ein wesentlicher Nachteil ist, dass trotz aller Maßnahmen, wie Vorbohren der Schraubenlöcher für die spätere Ostsynthese vor der eigentlichen Osteotomie, Einsatz einer zusätzlichen Schraube parallel zum Gelenksspalt, Durchtrennen des Knochens mit dem Meißel oder auch einer domförmigen Osteotomie, häufig kleine Stufen verbleiben, die als Risikofaktor für eine spätere Arthroseentwicklung angesehen werden. Dies wird umso deutlicher, je häufiger Schnittbildverfahren im weiteren Krankheitsverlauf angefertigt werden $[5,18]$.

Trotz aller operationstechnischen Herausforderungen finden sich in der Literatur gute Ergebnisse für die AMIC mit Innenknöchelosteotomie, ein direkter prospektiver randomisierter Vergleich ist bisher aber nicht erfolgt.

\section{Operativer Zugang ohne Innenknöchelosteotomie}

Aufgrund der beschriebenen Nachteile einer Innenknöchelosteotomie versuchen verschiedene Autorengruppen, den operativen Eingriff ohne Osteotomie durchzuführen. Hierzu wurden verschiedene Techniken entwickelt. Anhand anatomischer Studien konnte demonstriert werden, dass über eine entsprechende Wahl der Zugänge nahezu die gesamte Talusoberfläche ohne Osteotomie erreicht werden kann $[21,35]$. Die Möglichkeit, auf die Innenknöchel zu verzichten, bietet sich an, da zur Implantation einer AMIC kein Zugang im $90^{\circ}$ Winkel zur Knorpeloberfläche notwendig ist, wie z. B. bei der Implantation eines osteochondralen Stanzzylinders. Über den ventral-medialen Zugang, den ventral-zentralen Zugang sowie auch den ventral-lateralen Zugang lassen sich die ventralen zwei Drittel der Taluszirkumferenz gut erreichen. Der dorsale Sektor ist über einen dorsomedialen bzw. dorsolateralen Zugang besser zu adressieren [31]. 


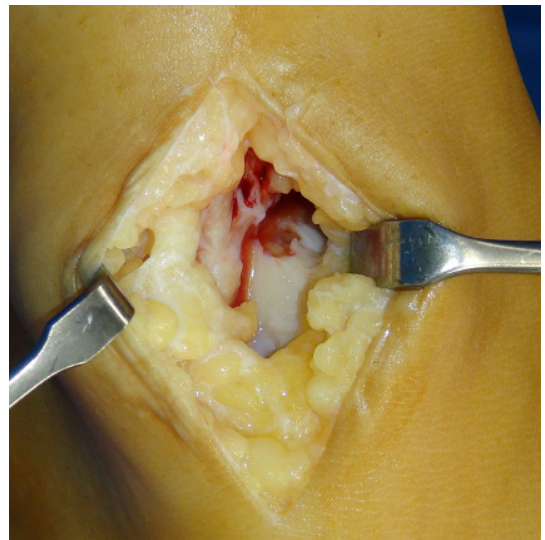

Abb. 1 ॥ Knorpelläsion an der medialen Taluskante des linken Sprunggelenks

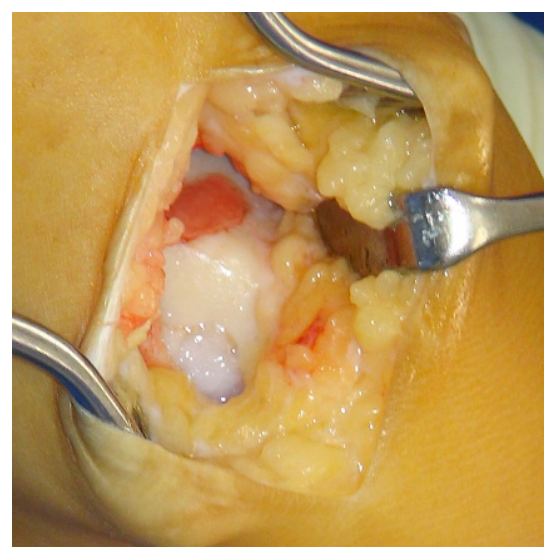

Abb. 4 \ Passgenaue Platzierung derChondroGide $^{\circledR}$-Membran (Geistlich Pharma AG, Wolhusen, Schweiz) in der Defektzone. Ein Überstand im Randbereich erhöht das Risiko für eine Delamination

\section{Arthroskopische Verfahren}

Grundsätzlich ist das Einbringen einer Kollagenmembran auch arthroskopisch möglich $[9,25,26]$. Voraussetzung ist, dass der Defekt arthroskopisch ausreichend gut erreicht wird. Limitierend können die knöchernen Veränderungen sein. Das Débridement größerer Knochenzysten gestaltet sich arthroskopisch oft schwierig, insbesondere wenn die Zystenwand vollständig entfernt werden soll. Auch kann sich bei größeren Zysten die arthroskopische Auffüllung schwierig gestalten. Einige Autoren empfehlen in dieser Situation lediglich eine Anbohrung von Zysten ohne Spongiosaplastik, unter der Vorstellung, dass sich die Zyste durch die Druckentlastung zurückbildet

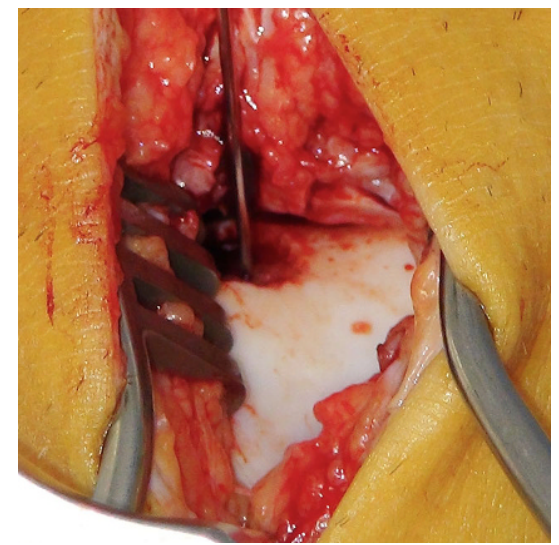

Abb. 2 - Anbohren von Sklerosezonen vor Auffüllung von Zysten mit Spongiosa, hier mit einem 0,8-mm-K-Draht

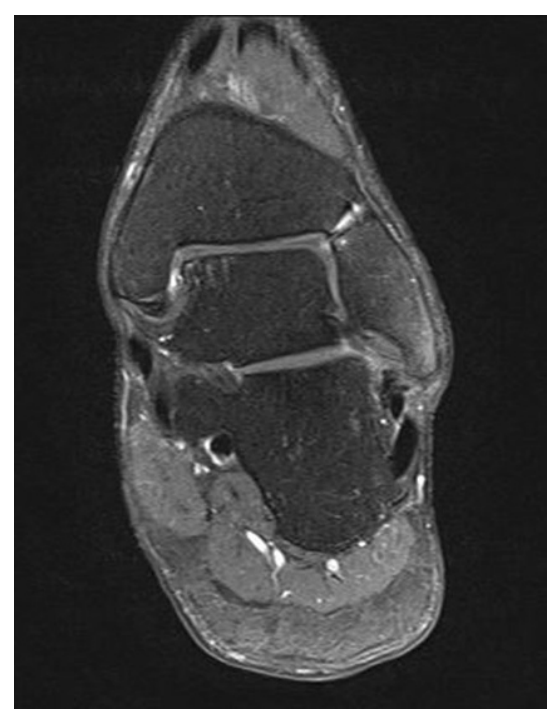

Abb. 5 ^ MRT (Magnetresonanztomographie) 24 Monate nach autologer matrixinduzierter Chondrogenese (AMIC). Ein diskretes Spongiosaödem unter der Membran ist noch erkennbar. Der Knorpelbelag zeigt sich durchgängig, die Spongiosaplastik ist gut eingewachsen

[31]. Die Größe des Defekts wird über die Referenz des Tasthakens abgeschätzt, dann wird das Sprunggelenk trockengelegt und die Membran eingebracht [25]. Die bisher publizierten Kurzzeitergebnisse sind vielversprechend, mittelfristige Ergebnisse stehen aber noch aus. Das arthroskopische Vorgehen unterscheidet sich ansonsten wenig von der offenen Technik.

\section{Operatives Vorgehen}

Der instabile Knorpel wird mit einer Kürette entfernt, bis zirkulär stabile Knor-

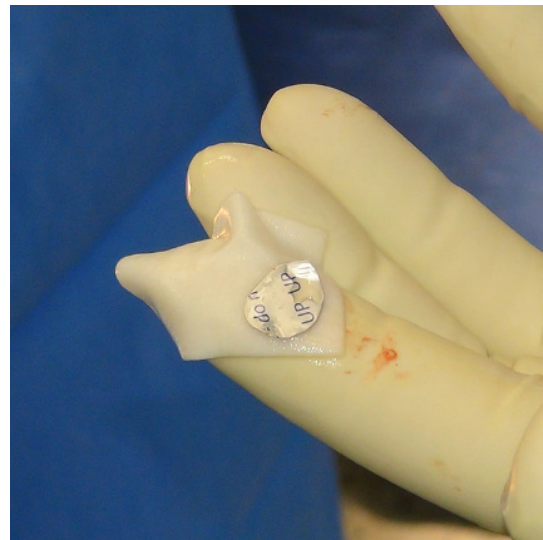

Abb. 3 A Übertragung der mit Alufolie bestimmten Größe auf die Chondro-Gide ${ }^{\circledR}$-Membran (Geistlich Pharma AG, Wolhusen, Schweiz). Zu beachten ist die dem Gelenk zugewandte und die dem Knochen aufliegende Seite

pelränder vorliegen ( $\bullet$ Abb. 1). Es hat sich bewährt, den Defekt rund oder ovalär zu präparieren, da dies das Einpassen der Membran deutlich erleichtert. Zysten am Talus werden vollständig ausgeräumt und die Zystenwand vollständig entfernt.

Anschließend erfolgt eine Mikrofrakturierung des subchondralen Knochens bzw. eine Anbohrung sklerosierter Zonen, wobei die Integrität der subchondralen Kortikalis als Auflagefläche möglichst erhalten werden sollte (• Abb. 2). Möglicherweise bietet die Nanofrakturierung Vorteile [3]. Mit einem speziellen Instrument werden Perforationen von mindestens $9 \mathrm{~mm}$ Tiefe gesetzt. Verglichen zur Mikrofakturierung konnte am Kniegelenkeine bessere Freisetzung von Stammzellen nachgewiesen werden [6]. Ein weiterer Vorteil ist, dass bei dieser Technik die subchondrale Knochenlamelle besser erhalten werden kann als bei der klassischen Mikrofakturierung. Am Talus liegen allerdings überwiegend osteochondrale Läsionen vor, so dass dieser theoretische Vorteil von geringer praktischer Relevanz ist.

Kleinere Defekte können gut mit Spongiosa vom gleichseitigen Kalkaneus oder von der distalen Tibia aufgefüllt werden [20].

Die Defektgröße kann nun anhand einer Aluminiumschablone bestimmt werden. Ist die Schablone passgenau zugeschnitten, wird die Größe auf die Membran übertragen. Die Membran kann trocken oder nach Befeuchtung 
mit physiologischer Kochsalzlösung zugeschnitten werden (• Abb. 3). Die feuchte Membran ist etwa 10-15\% kleiner als die trockene Membran, was beim Zuschneiden zu berücksichtigen ist. Das Zuschneiden der trockenen Membran ist einfacher in der Handhabung. Ziel ist es, die Membran passgenau zu präparieren, so dass diese ohne Überstand in den Defekt eingepasst werden kann (- Abb. 4). Nachdem die Membran auf den Defekt aufgelegt wurde, wird sie mit Fibrinkleber unterspritzt. Zusätzlich wird Fibrinkleber an den Randbereichen aufgetragen. Dies führt zu einer stabilen Fixation der Membran, auf eine Naht kann aus eigener Erfahrung verzichtet werden. Anschließend wird das Gelenk reponiert. Nach dem Aushärten des Fibrinklebers wird das Gelenk mehrfach durchbewegt. Überschüssiger Fibrinkleber wird entfernt. Zeigt die Membran beim Durchbewegen eine Delaminationstendenz, so ist entweder die Spongiosaplastik zu hoch oder es ist zu einer Überlappung der Membran in den Randbereichen gekommen. Je nach Ursache ist entweder die Größe der Membran nochmals anzupassen oder die oberflächliche Schicht der Spongiosaplastik abzutragen. Sobald die Membran stabil verankert ist, wird die Gelenkskapsel verschlossen. Bei trockenen Wundverhältnissen kann auf eine Drainage verzichtet werden. Postoperativ wird das Sprunggelenk für $48 \mathrm{~h}$ mit einer dorsalen Gipsschiene ruhiggestellt. Danach erfolgt der erste Verbandswechsel.

\section{Nachbehandlung}

Die Nachbehandlung umfasst eine Teilbelastung mit $10 \mathrm{~kg}$ für 6 Wochen an Unterarmgehstützen [1]. Bei ausgedehnter Spongiosaplastik ist in Einzelfällen auch eine vollständige Entlastung angezeigt. Im Anschluss daran erfolgt ein schmerzadaptierter Belastungsaufbau über weitere 2 bis 4 Wochen. Bis zur Vollbelastung ist eine Thromboseprophylaxe indiziert. Nach Abschluss der Wundheilung und Fadenzug, 14 Tage postoperativ, kann bei gut überdachten Läsionen auf eine weitere Gipsrückstellung verzichtet werden. Bewährt haben sich in dieser Situation Sprunggelenkor-

Arthroskopie 2018·31:111-116 https://doi.org/10.1007/s00142-017-0176-4

๑ Der/die Autor(en) 2017. Dieser Artikel ist eine Open-Access-Publikation.

M. Walther · A. Röser · H. Hörterer · A. Töpfer · A. Mehlhorn · O. Gottschalk

Autologe matrixinduzierte Chondrogenese am Sprunggelenk

\section{Zusammenfassung}

Während kleine osteochondrale Defekte am Talus gut durch die Mikrofrakturierung zu therapieren sind, bietet der Einsatz der autologen matrixinduzierten Chrondrogenese (AMIC) Vorteile in belasteten Zonen und bei Defekten $>1,5 \mathrm{~cm}^{2}$ - Situationen, in welchen für die Mikrofrakturierung weniger gute Ergebnisse beschrieben sind. In den publizierten Studien wurden jeweils knöcherne Defekte durch eine autologe Spongiosaplastik aufgefüllt und der instabile Knorpel débridiert. Die Abdeckung des Defekts erfolgte mittels einer Kollagenmembran, die Fixation mit Fibrinkleber. Das Verfahren ließ sich in der Mehrzahl der Fälle ohne Innenknöchelosteotomie durchführen. In Abhängigkeit von der Lokalisation wurden Membranen auch arthroskopisch unter Verzicht auf eine Arthrotomie ins Sprunggelenk eingebracht. MRT-Verlaufsstudien (Magnetresonanztomographie, MRT) zeigten, dass die Reparationsphase deutlich länger als 12 Monate andauert. Die publizierten Studien mit einem Nachuntersuchungszeitraum von 2 bis 3 Jahren berichteten über eine gute Schmerzreduktion mit einem AOFASScore (American Orthopedic Foot and Ankle Society, AOFAS) zwischen 80 und 90 Punkten. Erste Ergebnisse über einen 5-JahresNachuntersuchungszeitraum zeigten konstante Ergebnisse. Aktuell gibt es in der Literatur keine Evidenz, dass die wesentlich teurere und mit 2 Operationen aufwändigere autologe Chondrozytentransplantation der autologen matrixinduzierten Chondrogenese in der Therapie osteochondraler Läsionen am Talus überlegen ist.

\section{Schlüsselwörter}

Osteochondrosis dissecans - Osteochondrale Defekte · Talus · Knorpel · Rekonstruktion

\section{Autologous matrix-induced chondrogenesis of the ankle}

\section{Abstract}

While small osteochondral defects of the talus can be adequately treated by microfracturing, the use of autologous matrix-induced chrondrogenesis (AMIC) offers advantages in load-bearing zones and in defects larger than $1.5 \mathrm{~cm}^{2}$, situations where microfracturing has been proven to produce less favorable results. In the published studies, bony defects were filled with an autologous bone graft and the unstable cartilage was removed by debridement. The defect was then covered with a collagen membrane and the membrane was fixed with fibrin glue. In most of the cases, the procedure could be carried out without medial malleolar osteotomy. Depending on the localization, membranes were also implanted into the ankle joint by arthroscopy, with no need for arthrotomy. The magnetic resonance imaging (MRI) follow-up studies showed that regeneration lasted significantly longer than 12 months. The published studies with a follow-up of 2-3 years reported a satisfactory pain reduction with an American Orthopedic Foot and Ankle Society (AOFAS) score between 80 and 90 points. First results over a 5year follow-up period showed constant results. Currently, there is no evidence in the literature that the more expensive and with two operations more invasive autologous chondrocyte transplantation is superior to autologous matrix-induced chondrogenesis in the treatment of osteochondral lesions of the talus.

\section{Keywords}

Osteochondrosis dissecans · Osteochondral defect - Talus · Cartilage · Reconstruction thesen, die eine limitierte Dorsalextension und Plantarflexion ermöglichen, aber die Inversion und Eversion zuverlässig ausschalten. Ansonsten erfolgt die Rückstellung für 6 Wochen im UnterschenkelWalker, wobei auch hier mehrfach täglich das Sprunggelenk bewegt werden sollte.
Eine physiotherapeutische Unterstützung des Belastungsaufbaus und der Mobilisation des Gelenks mit Kräftigung der Muskulatur und Training der Propriozeption ist von Vorteil, Lymphdrainage erfolgt nach Bedarf.

Alltagsaktivitäten sind 8 bis 10 Wochen postoperativ meist möglich. Sport ohne Impaktbelastung, wie Fahrradfah- 
Tab. 1 Klinische Studien zur autologen matrixinduzierten Chondrogenese am Talus

\begin{tabular}{|c|c|c|c|c|c|c|c|c|c|c|c|}
\hline Autor & Jahr & Journal & $\begin{array}{l}\text { Evidenz- } \\
\text { level }\end{array}$ & $\begin{array}{l}\text { Verwendete } \\
\text { Membran }\end{array}$ & $\begin{array}{l}\text { FU } \\
\text { Mo }\end{array}$ & $n$ & AOFAS prä & $\begin{array}{l}\text { AOFAS } \\
\text { post }\end{array}$ & FFI prä & FFI post & $\begin{array}{l}\text { Mocart } \\
\text { Final FU }\end{array}$ \\
\hline $\begin{array}{l}\text { Giannini et al. } \\
\text { [12] }\end{array}$ & 2009 & $\begin{array}{l}\text { Clin Orthop } \\
\text { Relat Res }\end{array}$ & IV & Hyalograft & 29 & 48 & $46,4 \pm 15,5$ & $91,4 \pm 7,7$ & - & - & - \\
\hline $\begin{array}{l}\text { Valderrabano } \\
\text { et al. [28] }\end{array}$ & 2013 & $\begin{array}{l}\text { Am J Sports } \\
\text { Med }\end{array}$ & IV & Chondro-Gide & 31 & 26 & $\begin{array}{l}60 \\
\text { (R: 17-79) }\end{array}$ & $\begin{array}{l}89 \\
\text { (R: 61-100) }\end{array}$ & - & - & - \\
\hline $\begin{array}{l}\text { Giannini et al. } \\
\text { [11] }\end{array}$ & 2013 & $\begin{array}{l}\text { Am J Sports } \\
\text { Med }\end{array}$ & IV & $\begin{array}{l}\text { HYAFF- } 11^{\text {a }} \\
+ \text { Bone marrow } \\
\text { aspirate }\end{array}$ & 48 & 49 & $\begin{array}{l}63,73 \pm \\
14,13\end{array}$ & $\begin{array}{l}82,19 \pm \\
17,04\end{array}$ & - & - & - \\
\hline $\begin{array}{l}\text { Walther et al. } \\
\text { [32] }\end{array}$ & 2015 & ООТ & IV & Chondro-Gide & 34 & 14 & $50,14 \pm 9,8$ & $89,0 \pm 9,32$ & - & - & - \\
\hline $\begin{array}{l}\text { Kubosch et al. } \\
\text { [16] }\end{array}$ & 2015 & Int Orthop & IV & Chondro-Gide & 39,5 & 17 & - & $82,6 \pm 13,4$ & - & $\begin{array}{l}33,7 \pm \\
23,8\end{array}$ & $\begin{array}{l}52,7 \pm \\
15,9\end{array}$ \\
\hline $\begin{array}{l}\text { Wiewiorski } \\
\text { et al. [34] }\end{array}$ & 2016 & $\begin{array}{l}\text { Am J Sports } \\
\text { Med }\end{array}$ & IV & Chondro-Gide & 46 & 60 & $43 \pm 14$ & $76 \pm 17$ & - & - & - \\
\hline $\begin{array}{l}\text { Usuelli et al. } \\
\text { [27] }\end{array}$ & 2016 & Joints & IV & $\begin{array}{l}\text { Chondro-Gide } \\
\text { (Arthroskopisch) }\end{array}$ & 12 & 9 & $51,4 \pm 11,6$ & $71,8 \pm 20,6$ & - & - & $\begin{array}{l}51,7 \pm \\
16,6\end{array}$ \\
\hline $\begin{array}{l}\text { Usuelli et al. } \\
\text { [26] }\end{array}$ & 2016 & $\begin{array}{l}\text { Knee Surg } \\
\text { Sports Trau- } \\
\text { ma }\end{array}$ & IV & $\begin{array}{l}\text { Chondro-Gide } \\
\text { (Arthroskopisch) }\end{array}$ & 24 & 20 & $57,1 \pm 14,9$ & $86,6 \pm 10,9$ & - & - & $\begin{array}{l}50,9 \pm \\
24,9\end{array}$ \\
\hline $\begin{array}{l}\text { D'Ambrosi et al. } \\
\text { [9] }\end{array}$ & 2017 & Arthroscopy & IV & $\begin{array}{l}\text { Chondro-Gide } \\
\text { (Arthroskopisch) }\end{array}$ & 27 & 31 & $\begin{array}{l}53,03 \pm \\
15,60\end{array}$ & $\begin{array}{l}88,97 \pm \\
10,78\end{array}$ & - & - & - \\
\hline $\begin{array}{l}\text { Gottschalk et al. } \\
\text { [14] }\end{array}$ & 2017 & JFAS & III & Chondro-Gide & 60 & 21 & - & - & $56 \pm 18$ & $24 \pm 21$ & $54 \pm 14$ \\
\hline
\end{tabular}

ren und Schwimmen, können ab der 12. Woche begonnen werden. Die weitere Integration in den Sport gestaltet sich sehr unterschiedlich und ist neben Defektgröße und Lokalisation auch vom biologischen Regenerationsvermögen des individuellen Patienten abhängig [34]. Verlaufsuntersuchungen im MRT (Magnetresonanztomographen) haben gezeigt, dass die Regeneration des Knorpelgewebes 12 und mehr Monate in Anspruch nimmt, so dass Patienten auch nach einem Jahr auf intensive Belastung mit Beschwerden reagieren können ([16]; • Abb. 5).

\section{Ergebnisse}

Die Ergebnisse nach Rekonstruktion von Knorpeldefekten durch eine matrixinduzierte Chondrogenese liegen bei einem AOFAS-Score (American Orthopedic Foot and Ankle Society, AOFAS) von 80-90 Punkten [28, 32, 34]. Bei Ausgangswerten zwischen 50 und 60 Punkten bedeutet dies eine signifikante Verbesserung, allerdings ist auch ein AOFAS-Score von 90 Punkten weit von einem sportlich uneingeschränkt belastbaren Gelenk entfernt. Die genauere Analyse der Daten zeigt, dass die Verbesserung v. a. den Schmerz betrifft. Wierwiorsky et al. [34] untersuchten die Rückkehr zum Sport nach AMIC. Sie fanden, dass sich auch nach erfolgreicher Operation das sportliche Aktivitätsniveau im Vergleich zur Situation präoperativ nur geringfügig geändert hat. Als Ursachen werden möglicherweise noch vorhandene Restbeschwerden diskutiert, oder auch die Angst des Patienten, durch eine intensive sportliche Belastung das gute Operationsergebnis zu gefährden. Diese Zusammenhänge sollten beim Aufklärungsgespräch v. a. mit jungen und sportlich sehr aktiven Patienten sehr deutlich angesprochen werden. Das Verfahren ist nicht in der Lage, eine osteochondrale Verletzung rückgängig zu machen.

Einen wesentlichen Einfluss auf das Ergebnis scheint das verwendete Trägermedium zu haben (- Tab. 1). Die höchste Evidenz findet sich in der Literatur für Chondro-Gide $^{\circledR}$ (Geistlich Pharma AG, Wolhusen, Schweiz). Die Daten deuten darauf hin, dass die Regenerationsphase deutlich länger als 12 Monate anhält. So ist der Mocart-Score in den Langzeitergebnissen besser als in den Studien mit kurzer Nachuntersuchungsdauer [9, 14, 16, 25, 26]. Die Ergebnisse können nach heutigem Kenntnisstand nicht undifferenziert auf andere Produkte übertragen werden. So wurde für die Orthocell-Membran (Orthocell LTD, Australien) in einer kürzlich erschienenen Arbeit am Schafmodell (Kniegelenk) eine vermehrte Zystenbildung beschrieben [2].

\section{Diskussion}

Die Analyse der Daten zur Therapie von osteochondralen Läsionen am Sprunggelenk zeigt, dass sich bisher kein therapeutisches Verfahren als eindeutig überlegen durchgesetzt hat [1]. Die wissenschaftliche Evidenz ist für die Mikrofakturierung am höchsten, allerdings zeigen die Arbeiten auch klar die Grenzen der Mikrofakturierung, wie z. B. Defektgrößen $>1,5 \mathrm{~cm}^{2}$, stark belastete Zonen sowie die Verschlechterung der Ergebnisse nach 5 Jahren [7, 8, 17]. Die aktuelle Evi- 
denz zur AMIC, die inzwischen immerhin 5 Jahres-Ergebnisse umfasst, zeigt, dass mit der Verwendung von Kollagenmembranen in diesen Problemsituationen Ergebnisse erreicht werden können, wie sie sonst für die Mikrofakturierung im Allgemeinen beschrieben sind. Resultate mit einem AOFAS-Score deutlich über 90 Punkte erreicht keine der heute bekannten Techniken. Aufgrund klinisch vergleichbarer Resultate nach AMIC und nach Rekonstruktion von Knorpeldefekten mit einer autologen Chondrozytentransplantation wird heute der deutlich kostengünstigeren Membran der Vorzug gegeben.

\section{》) Die Behandlung osteo- chondraler Läsionen mit Kollagenmembranen ist eine Schmerztherapie}

Auch wenn fast alle Patienten über eine deutliche Schmerzlinderung berichten, lässt sich ein voll funktionsfähiges Sprunggelenk häufig nicht erreichen [34]. Dies betrifft insbesondere die hohe Erwartung an die sportliche Belastung. Hier ist unverändert ein realistisches Erwartungsmanagement Voraussetzung, um nicht mit einer großen Anzahl von enttäuschten Patienten konfrontiert zu werden.

Die Behandlung osteochondraler Läsionen mit Kollagenmembranen ist in erster Linie eine Schmerztherapie. Bei Patienten mit wenig Beschwerden und guter sportlicher Belastbarkeit ist daher die Indikation zur Operation sehr kritisch zu stellen, da möglicherweise ein operativer Eingriff keine für den Patienten wahrnehmbare Verbesserung der Situation ergibt. Eine MRT-Verlaufskontrolle in 12 Monaten sowie die Empfehlung einer Konsultation bei zunehmenden Beschwerden ist für solche Patienten häufig die bessere Therapie - dies gilt umso mehr, da bisher keine Studie belegt hat, dass es sich bei der Osteochondrosis dissecans um eine Präarthrose handelt, die zwingend einen prophylaktischen operativen Eingriff rechtfertigen würde [10].

Die heute verfügbaren Therapieoptionen sind definitiv nicht das Ende der Entwicklung. Aktuelle Forschungen beschäftigen sich v. a. mit der intraoperativen Beschichtung der Membran mit Stammzellen und der Applikation von Wachstumsfaktoren in der postoperativen Phase [4, 29, 30]. Es bleibt abzuwarten, mit welchem Verfahren es gelingen wird, die magische Grenze von 85-90 Punkten im AOFAS-Score zu durchbrechen.

\section{Fazit für die Praxis}

- Die autologe matrixinduzierte Chrondrogenese ermöglicht die Rekonstruktion größerer osteochondraler Defekte am Talus.
- Kollagenmembranen bieten v. a. in belasteten Zonen und bei Defekten $>1,5 \mathrm{~cm}^{2}$ Vorteile.

- Die Regenerationsphase dauert länger als 12 Monate.

- Die Ergebnisse zeigen über einen 5-Jahres-Nachuntersuchungszeitraum konstante Ergebnisse.

- Das Verfahren lässt sich in der Mehrzahl der Fälle ohne Innenknöchelosteotomie durchführen.

- Kleinere Defekte mit geringer ossärer Beteiligung können arthroskopisch therapiert werden.

- Aktuell gibt es keine Evidenz, dass die wesentlich teurere und mit 20 perationen aufwändigere autologe Chondrozytentransplantation der autologen matrixinduzierten Chondrogenese überlegen ist.

\section{Korrespondenzadresse}

\section{Prof. Dr. med. M. Walther}

Zentrum für Fuß- und Sprunggelenkchirurgie, Schön Klinik München Harlaching, FIFA Medical Centre

Harlachinger Straße 51, 81547 München, Deutschland

mwalther@schoen-kliniken.de

\section{Einhaltung ethischer Richtlinien}

Interessenkonflikt. M. Walther und O. Gottschalk geben an, dass sie in Workshops zur Knorpelrekonstruktion tätig waren, die teilweise von im Artikel genannten Firmen finanziell unterstützt wurden. A.

Hier steht eine Anzeige. 
Röser, H. Hörterer, A. Töpfer und A. Mehlhorn geben an, dass kein Interessenskonflikt besteht.

Dieser Beitrag beinhaltet keine von den Autoren durchgeführten Studien an Menschen oder Tieren.

Open Access. Dieser Artikel wird unter der Creative Commons Namensnennung 4.0 International Lizenz (http://creativecommons.org/licenses/by/4.0/deed. de) veröffentlicht, welche die Nutzung, Vervielfältigung, Bearbeitung, Verbreitung und Wiedergabe in jeglichem Medium und Format erlaubt, sofern Sie den/die ursprünglichen Autor(en) und die Quelle ordnungsgemäßnennen, einen Link zur Creative Commons Lizenz beifügen und angeben, ob Änderungen vorgenommen wurden.

\section{Literatur}

1. Aurich M, Albrecht D, Angele $P$ et al (2017) Treatment of Osteochondral lesions in the ankle: a guideline from the group "clinical tissue regeneration" of the German Society of Orthopaedics and Traumatology (DGOU).ZOrthop Unfall 155:92-99

2. Beck A, Murphy DJ, Carey-Smith R et al (2016) Treatment of articular cartilage defects with microfracture and autologous matrix-induced chondrogenesis leads to extensive subchondra bone cyst formation in a sheep model. Am J Sports Med 44:2629-2643

3. Benthien JP, Behrens P (2015) Nanofractured autologous matrix induced chondrogenesis (NAMIC(c)) - further development of collagen membrane aided chondrogenesis combined with subchondral needling: a technical note. Knee 22:411-415

4. Buda R, Vannini F, Cavallo M et al (2013) Onestep bone marrow-derived cell transplantation in talarosteochondral lesions: mid-term results. Joints 1:102-107

5. Bull PE, Berlet GC, Canini C et al (2016) Rate of malunion following bi-plane chevron medial malleolar osteotomy. Foot Ankle Int 37:620-626

6. Chen H, Hoemann CD, Sun J et al (2011) Depth of subchondral perforation influences the outcome of bone marrow stimulation cartilage repair. JOrthop Res 29:1178-1184

7. Choi WJ, Kim BS, Lee JW (2012) Osteochondral lesion of the talus: could age be an indication for arthroscopic treatment? Am J Sports Med 40:419-424

8. Chuckpaiwong B, Berkson EM, Theodore GH (2008) Microfracture for osteochondral lesions of the ankle: outcome analysis and outcome predictors of 105 cases. Arthroscopy 24:106-112

9. D'ambrosi R, Maccario C, Serra N et al (2017) Osteochondral lesions of the talus and autologous matrix-induced chondrogenesis: Is age a negative predictor outcome? Arthroscopy 33:428-435

10. Elias I, Jung JW, Raikin SM et al (2006) Osteochondrallesions of the talus: change in MRI findings over time in talar lesions without operative intervention and implications for staging systems. Foot Ankle Int 27:157-166

11. GianniniS, Buda R, Battaglia Metal (2013)One-step repair in talar osteochondral lesions: 4-year clinical results and t2-mapping capability in outcome prediction. Am J Sports Med 41:511-518

12. Giannini S, Buda R, Vannini F et al (2009) Onestep bone marrow-derived cell transplantation in talar osteochondral lesions. Clin Orthop Relat Res 467:3307-3320

13. Gierloff M, Nitsche T, Adam-Klages S et al (2014) In vitro comparison of different carrier materials with rat bone marrow MSCs. Clin Oral Investig 18:247-259

14. Gottschalk O, Altenberger $S$, Baumbach $S$ et al (2017) Functional medium-term results after autologous matrix-induced chondrogenesis for osteochondral lesions of the talus: a 5-year prospective cohort study. J Foot Ankle Surg. https://doi.org/10.1053/j.jfas.2017.05.048

15. Hunt KJ, Lee AT, Lindsey DP et al (2012) Osteochondral lesions of the talus: effect of defect size and plantarflexion angle on ankle joint stresses. Am J Sports Med 40:895-901

16. KuboschEJ, ErdleB, Izadpanah Ketal (2016)Clinical outcome and $\mathrm{T} 2$ assessment following autologous matrix-induced chondrogenesis in osteochondral lesions of the talus. Int Orthop 40:65-71

17. Lee KB, Bai LB, Yoon TR et al (2009) Second-look arthroscopic findings and clinical outcomes after microfracture for osteochondral lesions of the talus. Am J Sports Med 37(Suppl 1):63S-70S

18. Leumann A, Horisberger M, Buettner O et al (2016) Medial malleolar osteotomy for the treatment of talar osteochondral lesions: anatomical and morbidity considerations. Knee Surg Sports Traumatol Arthrosc 24:2133-2139

19. Loveday D, Clifton R, Robinson A (2010) Interventions for treating osteochondral defects of the talus in adults. Cochrane Database Syst Rev. https://doi. org/10.1002/14651858.CD008104.pub2

20. Miller CP, Chiodo CP (2016) Autologous bone graft in foot and ankle surgery. Foot Ankle Clin 21:825-837

21. Muir D, Saltzman CL, Tochigi Y et al (2006) Talar dome access for osteochondral lesions. Am J Sports Med 34:1457-1463

22. Murawski CD, Kennedy JG (2013) Operative treatment of osteochondral lesions of the talus. JBone Joint Surg Am 95:1045-1054

23. Pot MW, Gonzales VK, Buma Petal (2016) Improved cartilage regeneration by implantation of acellular biomaterials after bone marrow stimulation: a systematic review and meta-analysis of animal studies. PeerJ 4:e2243

24. Richter W (2009) Mesenchymal stem cells and cartilage in situ regeneration. J Intern Med 266:390-405

25. Usuelli FG, De Girolamo L, Grassi Met al (2015) Allarthroscopic autologous matrix-induced chondrogenesis for the treatment of osteochondral lesions of the talus. Arthrosc Tech 4:e255-e259

26. Usuelli FG, D'ambrosi R, Maccario C et al (2016) All-arthroscopic AMIC(R) (AT-AMIC(R)) technique with autologous bone graft for talar osteochondral defects: clinical and radiological results. Knee Surg Sports Traumatol Arthrosc. https://doi.org/ 10.1007/s00167-016-4318-4

27. Usuelli FG, Grassi M, Manzi L et al (2016) Treatment of osteochondral lesions of the talus with autologous collagen-induced chondrogenesis: clinical and magnetic resonance evaluation at oneyear follow-up. Joints 4:80-86

28. Valderrabano V, Miska M, Leumann A et al (2013) Reconstruction of osteochondral lesions of the talus with autologous spongiosa grafts and autologous matrix-induced chondrogenesis. Am J Sports Med 41:519-527

29. Vannini F, Di Matteo B, Filardo G (2015) Platelet-rich plasma to treat ankle cartilage pathology - from translational potential to clinical evidence: a systematic review. J Exp Orthop 2:2
30. Vannini F, Cavallo M, Ramponi L et al (2017) Return to sports after bone marrow-derived cell transplantation for osteochondral lesions of the talus. Cartilage 8:80-87

31. Walther M, Becher C, Volkering C et al (2012) Treatment of chondral and osteochondral defects of the talus by autologous matrix induced chondrogenesis. Fuss Sprunggelenk 10:121-129

32. Walther M, Altenberger S, Kriegelstein Set al (2014) Reconstruction of focal cartilage defects in the talus with miniarthrotomy and collagen matrix. Oper Orthop Traumatol 26:603-610

33. Wiewiorski M, Barg A, Valderrabano V (2013) Autologous matrix-induced chondrogenesis in osteochondral lesions of the talus. Foot Ankle Clin 18:151-158

34. Wiewiorski $M$, Werner L, Paul J et al (2016) Sports activity after reconstruction of osteochondral lesions of the talus with autologous spongiosa grafts and autologous matrix-induced chondrogenesis. Am J Sports Med 44:2651-2658

35. Young KW, Deland JT, Lee KT et al (2010) Medial approaches to osteochondral lesion of the talus without medial malleolar osteotomy. Knee Surg Sports Traumatol Arthrosc 18:634-637 